\title{
Il piano regolatore di Tripoli (1930-1936). La consapevolezza del passato
}

Tripoli town plan (1930-1936). The consciousness of the past

\author{
Michela Marisa Grisoni \\ Politecnico di Milano, Milan, Italy, michela.grisoni@polimi.it
}

\begin{abstract}
The paper recalls the well known urban facts of Tripoli during the Italian colonialism to eventually deepen the theme of the preservation of the past and not only of the Roman one, as well of the city walls. The town plan has been analyzed not only as it has been approved but also as it has been argued, not only through the drawings but also by the debate. A few letters between the professionals involved (especially Alberto Alpago Novello) and some authoritative exponents of the contemporary architecture culture and criticism (like, Gustavo Giovannoni) have assured an original source to underlines the critical background and to reveal a purpose of touristic and commercial development.
\end{abstract}

Keywords: Italian colonialism, city-walls, Alpago Novello, Cabiati, Marelli.

\section{Antefatti}

Un secolo fa Tripoli si progettava non solo capitale della Tripolitania ma città turistica ${ }^{1}$. Italiana dal $1911^{2}$, era stata oggetto di un rilievo topografico (1912) propedeutico alla stesura di un piano regolatore (1914) concepito dai tecnici del Genio Civile. Si era già innescata una massiccia attività edilizia e mentre la "città nuova" si era estesa lungo alcuni assi principali, si propagandava la conservazione del "tessuto edilizio antico" posto all'interno delle mura e ritenuto intatto. Per alcuni la suddivisione era accentuata, più che dalle mura, già in parte atterrate, dal forte spagnolo e dal castello. Assunti a cardini della struttura urbana, queste antiche "difese" parevano strumenti, cerniere fisiche ma anche ideali, per separare il passato dal presente della città (Marchetti, 1931; Ornato, 1932).

Altri illustravano una forma urbana più ripartita. Spiegavano che Tripoli aveva il suo volto vecchio e quello nuovo ma che se ne progettava anche uno nuovissimo. A sud-ovest della città vecchia, oltre il "recinto" delle mura, il tracciato di tre nuove strade disegnava "un triangolo", ovve- ro una "zona" "destinata ad essere il centro della città europea futura"; tale si prefigurava Tripoli nelle guide (Tripoli, dintorni, 1914).

\subsection{Escursione a Tripoli (1914)}

Compilata nel febbraio del 1914 sotto la direzione del Capo dell'Ufficio Cartografico del Ministero delle Colonie ma frutto di un lavoro condiviso, la guida Tripoli e dintorni era destinata ai partecipanti alla Escursione Nazionale in Tripolitania organizzata dal Touring Club Italiano in quello stesso anno.

Tre le cartografie di corredo. A quelle più convenzionali, per tema e scala, destinate a rappresentare l'intera regione (1:500000) e la capitale (1:5000) e rilegate in fondo al volume, se ne sommava infatti una terza, più piccola e intercalata alle pagine.

Rappresentava la planimetria della città ad una scala tale (1:15000) da includere non soltanto la parte vecchia e quella nuova ma, per l'appunto, 


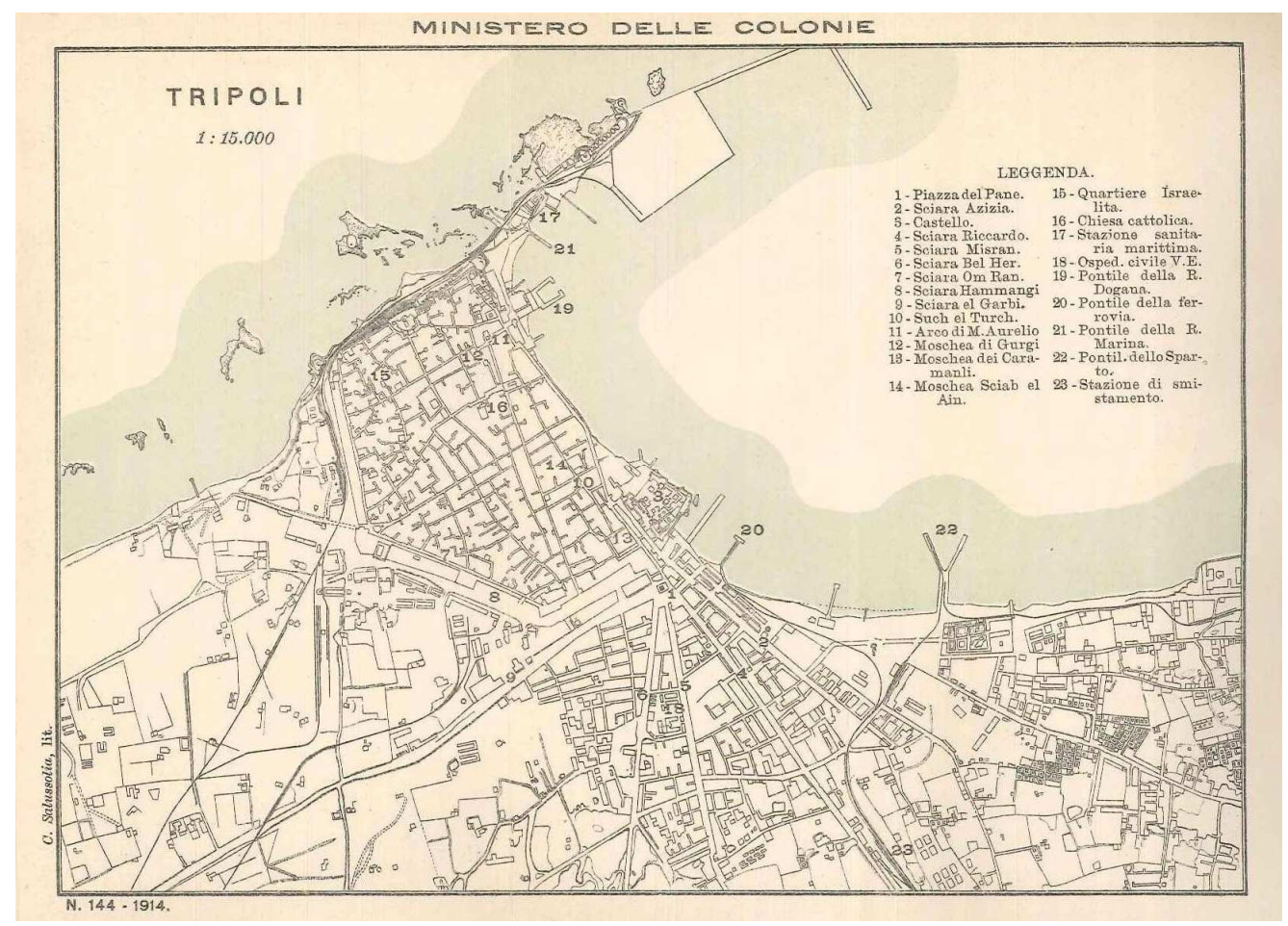

Fig. 1. Pianta di Tripoli nel 1914 (Tripoli e dintorni, 1914).

anche quelle di ampliamento, a sud-ovest, previste dal Piano Regolatore (Fig. 1). Fattore di scala e modalità di presentazione facilitavano la lettura del piano (delineato al tratto e stampato in rosso su velina a parte da sovrapporre alla planimetria dello stato di fatto) (Fig. 2). Chiaro comprendere che le aree di ampliamento previste erano in verità tre: oltre al citato triangolo, si prevedeva una vasta zona industriale, a ovest, all'estremità opposta e ugualmente lungo la costa, quella dei "villini”. La guida era un omaggio da distribuire agli escursionisti che si auspicava di coinvolgere nel progetto di "europeizzazione" della città. Nel didascalico testo emergevano le storie di successo degli immigrati italiani in Tripolitania. Si tentava cioè di eccitare altre iniziative, assecondando una dinamica migratoria già in atto ed anteriore agli eventi del 1911. Alcune immagini mostravano una comunità artigianale operosa dedita alla tintoria, alla tessitura o all'oreficeria, alla lavorazione di pellame o piume di struzzo. Si palesava la relazione di reciprocità esistente tra "gli indigeni", le loro attività e i luoghi deputati a compierle. Forte dell'immediatezza del mezzo fotografico, l'analisi urbana era lettura sincronizzata dell'episodio architettonico e della società che vi abitava e lavorava. Che fosse attraverso la fotografia di un pozzo, l'interno di un laboratorio o lo scorcio di una strada fitta di botteghe, il lettore era sollecitato a vedere luoghi abitati, a cogliere le astuzie locali del costruire: i prospetti ciechi (a proteggere l'intimità della casa araba), le strade tortuose (a difendere i passanti dal ghibli) e coperte (a proteggerli dalla calura), i vicoli ciechi (ad isolare gli ambiti privati entro il fitto tessuto edilizio).

Nonostante l'articolata visione del fenomeno urbano e umano come commistione di elementi culturali tra di loro anche molto eterogenei (turchi, arabi, israeliti e romani) il "Cicerone" del Ministero delle Colonie tradiva accenti nazionalisti e fini propagandistici. E' palese che nella Guida l'interesse per la cultura locale scivola nel regionalismo più pittoresco con equivoci, anche gravi, sulla storia. Non un cenno al servizio di tutela, strutturato quello stesso anno (R. Decreto 24 settembre 1914, n. 1271), pochi i monumenti segnalati, prediligendo quelli di età romana più eclatanti e i reperti musealizzabili. 


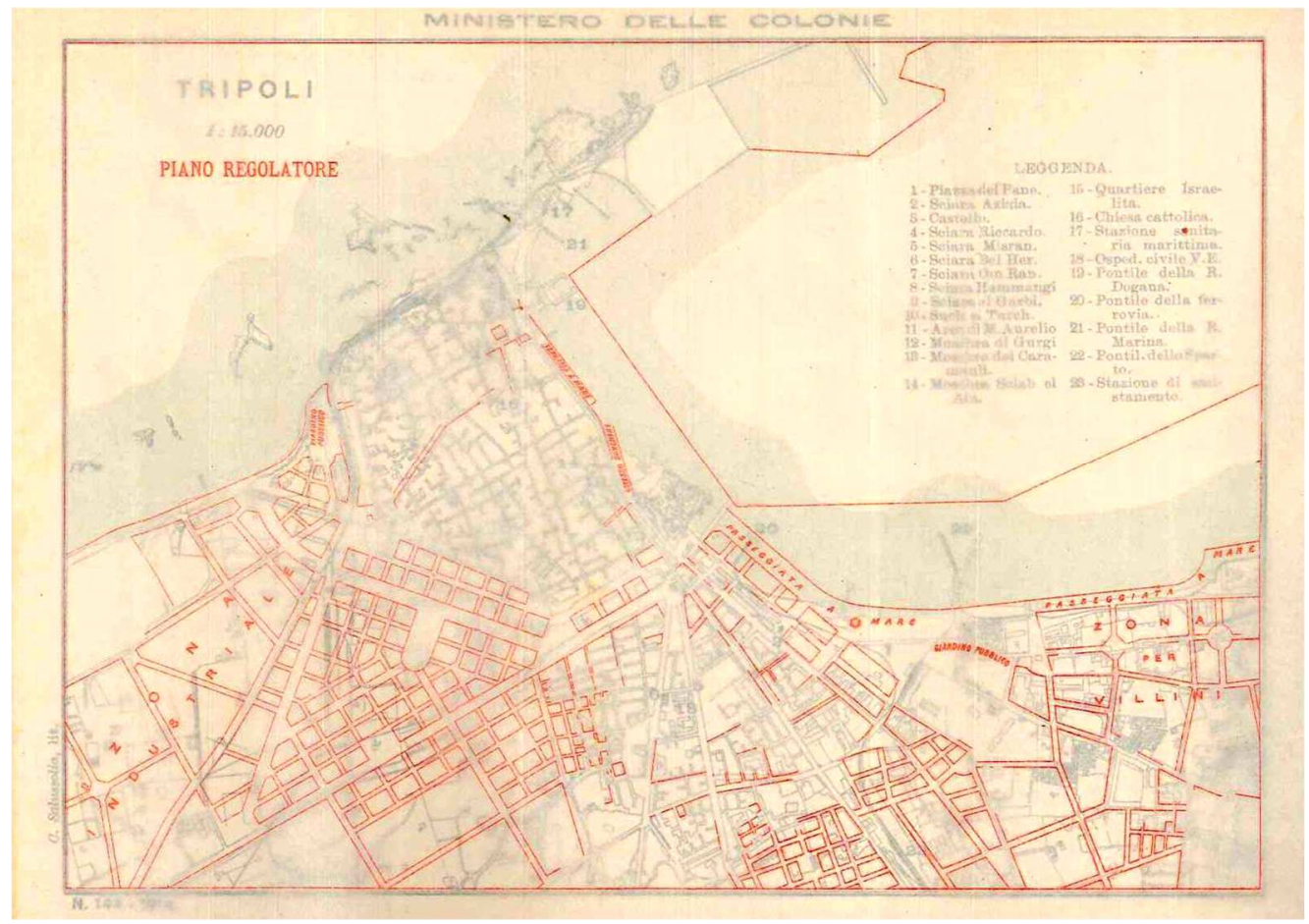

Fig. 2. Piano regolatore di Tripoli 1914 (Tripoli e dintorni, 1914). Al centro il triangolo della città nuovissima, a sinistra la zona industriale, a destra quella dei villini.

Riduttivo il riferimento al castello (destinato a sede istituzionale e museo); frettoloso quello al forte spagnolo; trascurati i fondachi e ignorate anche le mura, peraltro equivocamente dette romane e ridotte ad una impronta a terra da ricalcare con una nuova strada e nuove costruzioni. Il piano regolatore ne decretava infatti la demolizione. Lo mostra la planimetria e lo rimarcherà Pietro Romanelli biasimando l'atto perché indiscriminato, disarticolato e pretestuoso (Romanelli, 1923).

\section{2. "Una sentenza capitale pronunciata senza giudizio e con procedimento sommario"}

Archeologo allievo, tra gli altri di Emanuele Löwy, perfezionatosi alla Scuola italiana di archeologia di Atene, di cui seguì le missioni in Asia Minore condotte da Roberto Paribeni, Pietro Romanelli (1889-1981) era stato reclutato dall'apparato statale di tutela e assegnato ai servizi archeologici di Libia nel 1915. Nel 1918 subentrò nel ruolo di Soprintendente a Salvatore Aurigemma (1885-1969).
A sua volta questi, condotto in Cirenaica e Tripolitania da Federico Halbherr nel 1911, era stato tra i protagonisti della prima fase di esplorazione delle regioni e l'autore di apprezzabili indagini sul campo (Mariani, 1915; Aurigemma, 1930). Operò, suo malgrado, al servizio di un governo incline a strumentalizzare il passato romano dell' Africa mediterranea per giustificare la propria politica coloniale. Di contro egli dimostrò precocemente $\mathrm{e}$ in più occasioni una sensibilità per altre fasi della storia d'Africa (Aurigemma, 1915).

\subsection{Il restauro degli avanzi delle mura bar- baresche. 1921-1922}

Esperto romanista, giunto a Tripoli, Pietro Romanelli ricalcò le orme di Aurigemma. Nel 1923, mentre stigmatizzava la demolizione delle mura "atterrate" per il prevalere di un "fervore innovativo', illustrava gli esiti di un rilievo, debitore degli studi del suo predecessore ma in parte personalmente condotto, del complesso e pluri stratificato, sistema 'difensivo' della città. Lasciava così emergere la piena assimilazione di una metodologia di studio del passato che, ricor- 
rendo tanto alle fonti dirette che a quelle indirette, considera il monumento stesso come documento e di necessità avverte l'opportunità di salvaguardarne l'autenticità. Emergeva anche un atteggiamento di diffidenza nei confronti delle datazioni basate sulla sola lettura dei caratteri costruttivi o stilistici, spesso ricorrenti o reiterati e dunque cronologicamente poco circoscritti cui, di contro, opponeva la lettura dei rapporti stratigrafici, rivelando un'attenzione non comune non tanto per la stratigrafia dei suoli quanto per quella degli elevati. Tale approccio è tanto più significativo perché propedeutico al restauro, compiuto tra il 1921 e il 1922, di quanto era sopravvissuto, dopo le demolizioni, peraltro più estese del previsto. Due le questioni affrontate: la conservazione degli spezzoni di muratura rimasti e il palesarsi di fasi appartenenti ad epoche distinte (Fig. 3).
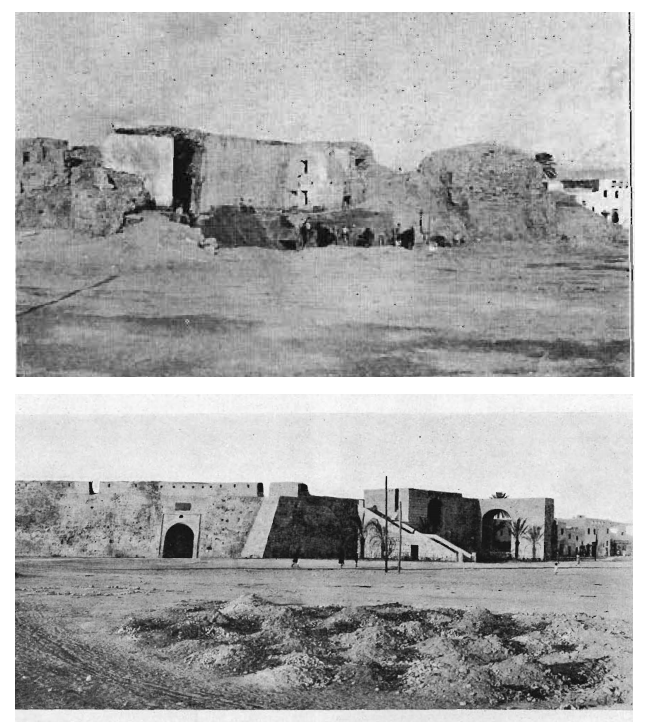

Fig. 3. L'arco di Bâb Zanâta prima dei restauri, sopra, e dopo, sotto (Romanelli, 1923).

Eseguì i necessari consolidamenti e respinse le ricostruzioni analogiche, assimilando terminologie e categorie di intervento oramai codificate; acconsentì alle ricostruzioni se funzionali al riuso ed ammise la ricerca di accordi armonici tra le parti se pure nel rispetto dei rapporti stratigrafici tra le stesse.

Nel corso del rilievo e del restauro, ipotizzò alcune cronologie relative ed escluse che fossero sopravvissute costruzioni di età romana o bizantina pur ammettendo, per esempio nelle cortina a conci, che vi fossero elementi di reimpiego di quelle epoche (Romanelli, 1923): acquisizione originale, tanto più autorevole perché espressa da un romanista che non mercificò le proprie competenze per fini politici.

\section{Verso un nuovo piano regolatore}

Romanelli lasciò Tripoli nel 1925, assegnato al Museo Nazionale Romano. La sua presenza fu breve e per alcuni aderente "alle direttive ideologiche del regime fascista" (Vistoli, 2017); ma forse il giudizio va sgravato dai carichi portati e ricalibrato sui suoi soltanto. Illustrando i restauri delle mura di Tripoli l'archeologo, per esempio, distinse chiaramente la romanità autentica da quella ideale e non lesinò critiche per le ulteriori demolizioni della città antica previste dal piano regolatore.

\subsection{I progetti libici di un trio di architetti}

Per il trio Alberto Alpago Novello (1889-1985), Ottavio Cabiati (1889-1956) e Guido Ferrazza (1887-1961) gli incarichi libici si sarebbero profilati incidentalmente, favoriti da contatti e relazioni, non soltanto professionali, coltivati dall'ultimo dei tre, in particolare (Zanella 2002). A Tripoli intervennero i primi due soltanto sul vigente, citato e criticato, Piano Regolatore, modificandolo già nel 1930. Si trattava di un tema di progettazione urbana: non il primo affrontato, nè in Italia (si rammentino quelli di Verona e Pisa e, ovviamente, il concorso per il Piano Regolatore della Città Milano) nè Oltremare. Lo precede quello per Bengasi, affidato però al trio.

Poche le fonti conservate nel loro archivio: carteggi e qualche tavola (Zanella, 2002). Il lavoro associato e la successiva rottura del sodalizio, l'avvio di altre collaborazioni e la stessa drammatica biografia di alcuni, oltre alla usuale avventurosa conservazione degli archivi, ne hanno condizionato e sparpagliato la consistenza (Zanella, 1991; Grisoni, 2016). Se pure frammentaria, la 'documentazione di lavoro' sulla Cirenaica e su Tripoli già edita comprende alcune decine di fotografie tra cui quelle sulle rovine di Cirene o, le altre, meno scontate sulle case arabe.

Di Tripoli colpiscono quelle raffiguranti le porte di ingresso alla Medina: possibile indizio del loro interesse per le "connessioni" tra la città vecchia e la sua espansione, oltre le mura. Quanto la regione e la città abbiano impressionato i due architetti è del resto documentato anche dai vi- 
branti colori dei loro acquarelli (Fig. 4), dalla spontaneità degli appunti, dalla sincerità della corrispondenza con i familiari.

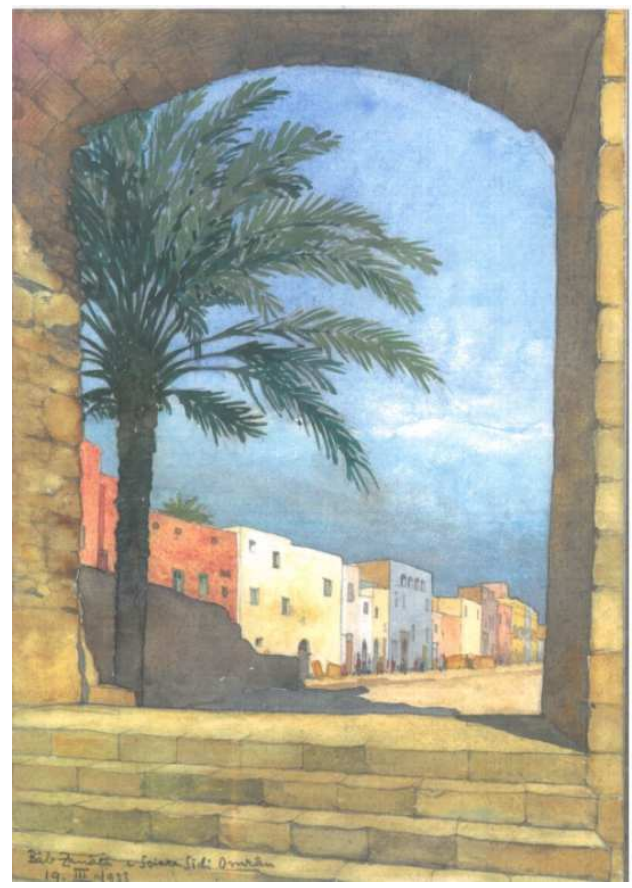

Fig. 4. L'arco di Bâb Zanâta dopo i restauri in un acquerello di Alberto Alpago Novello (Collezione privata).

In un genuino ed autografo Memoriale, Alpago Novello confida il Mal d'Africa provato "p. es. arrivando con Cabiati a Cirene -bloccati a mezzavia da una violenta burrasca, e rifugiatisi dentro un (conservatissimo) mausoleo romano- per quel mare azzurro cupo, e il sole a picco, e quelle distese desertiche -oppure anche uno sconfinato ondeggiar di frumento-". Simili tonalità rese Cabiati nei suoi acquarelli; tre quelli tripolini pubblicati (Fabiani, 1991). Altrettanto istantanei gli schizzi e le misure annotate ai margini delle cartoline postali (Zampagni, 2006).

\subsection{Un nuovo piano di espansione}

Il compito loro assegnato riguardava le aree esterne alle mura: già interessate dall'espansione edilizia ora erano definitivamente destinate al disegno della città nuova. Nella loro proposta è apparso preminente il tracciato della rete viaria (Zanella, 2002). In effetti si trattava di assicurare uno scorrimento fluido e un collegamento age- vole tra le diverse parti della capitale sul modello delle città europee più moderne cui loro stessi più volte ammisero di riferirsi. La nervatura pensata per circolare all'interno dell'organismo urbano sembra effettivamente ancorarsi a taluni punti notevoli e definire, di risulta, gli isolati: da edificare se liberi, da conservare se a carattere monumentale, da ricucire se disarticolati rispetto ad un sistema urbano che si voleva funzionale e integrato. Se non il piano, alla cui messa a punto concorsero una varietà di ragioni e impulsi, la riflessione degli architetti pare però più inclusiva.

Nella città di espansione si prefiguravano aree distinte: sei, in particolare, i quartieri periferici progettati per ricucire l'area nord-ovest. Si richiedeva inoltre, programmaticamente, di fronteggiare l'eccessiva densità abitativa della città entro le mura. Per tentare di ridurla si concepì un nuovo quartiere arabo, esterno ad esse, nell'area prospettante il lungomare della Vittoria, ribassata alla scopo. La cesura tra centro antico e area di nuova espansione fu così ridiscussa. Le mura non furono adottate come un alibi, ricorrente ed abusato, per separare l'antico, o isolare i residenti.

Né soglia, né limite, né confine, le mura, o quel che ne restava, per taluni erano piuttosto incluse in un progetto di valorizzazione, anche a fini turistici, dei percorsi pedonali connessi ai nuovi viali lungomare (ivi compreso quindi il nuovo quartiere arabo). Si innescò cioè una riflessione sull'opportunità e sulle modalità di intrecciare genti, culture, architetture tanto differenti, "mitigandone" i possibili contrasti. Si potrà obiettare che la creazione di un quartiere arabo dedicato tendesse capziosamente ad espellere gli indigeni dal centro smorzando gli accenti positivi avvertiti nella loro proposta. Ma tra le tante idee raccolte è legittimo credere che ve ne fosse davvero una pacifica in base alla quale, storicità e tradizioni 'indigene' non dovessero essere confinate entro il recinto murario della "Medina"; che si potesse progettare la permeabilità della città antica attraverso lo studio delle visioni prospettiche ed innescare uno sviluppo più organico dell'ambiente urbano; anche se non tutta la storiografia riconosce un significato esemplare al piano.

\section{Gli elementi caratteristici della città}

Proprio di "ambiente" parlava infatti Michele Marelli per spiegare i criteri cui si era ispirato per la "sistemazione" dell' Arco di Marco Aure- 
lio (Marelli, 1933). E' già stato rilevato che, a fianco dell'incarico per la redazione del piano, correvano quelli dedicati alla progettazione, o sistemazione, di singoli edifici o zone della città. Un decennio prima un restauro era stato finalizzato a liberare, isolare e ripristinare il monumentale arco (Boni-Mariani, 1915). Nel 1931, mentre se ne sollecita il consolidamento (Aurigemma, 1931), l'incarico riguarda la sistemazione dell'ambiente circostante. D'intesa con la Soprintendenza della Tripolitania, Marelli scartò le proposte precedentemente prefigurate da Giacomo Boni (1859-1925) tendenti a riprendere gli sbancamenti utili sia ad un più esteso isolamento che alla ricerca dei "frammenti" dell' arco ancora mancanti ${ }^{3}$. Disapprovò anche la demolizione dei "tre fonduchi maggiori, già "condannati alla distruzione". Se pure "accessorio" rispetto al compito di restituire "preponderanza" e "respiro" al "Monumento", si pose cioè anche l'obbiettivo di preservare elementi della città meno altisonanti ma molto caratteristici, quali, appunto, $i$ fondachi. Li sottopose quindi a demolizioni selettive, valutando le visuali possibili in rapporto alla disposizione e all'orientamento dell'arco ed ai risanamenti atti a soddisfare basilari parametri igienici. Ne rimodellò le forme per ottenere avvaloramenti reciproci, in modo da "costruire" un insieme armonico in cui i fondachi mantenuti all'uso di residenze, avrebbero potuto ospitare anche botteghe, piccole industrie, laboratori artigiani come istituti, scuole ed uffici. Come altri, intravedeva nella città storica una pluralità di attrattive non solo romane, con opportunità di promozione del turismo. Definì distanze, allontanamenti e avvicinamenti tra gli edifici. Studiò le quote dei piani di calpestio progettando accessi per ovviare al notevole abbassamento di quello romano. Dispose opportunamente aiuole e alberature. Rinunciò ad ulteriori sterri che avrebbero accentuato il dislivello, ma si assicurò di evidenziare la funzione del monumento rispetto all'incrocio di cardo e decumano.

Curò le visuali prospettiche, ma non cedette alle lusinghe dei rettifili monumentali, a scenografici sventramenti. Preferì seguire tracciati "spontanei e di minor percorso", accettò le visuali interrotte. Favorì la permeabilità ricavandola tra la diacronia delle scenografie urbane. Era consapevole che vi fossero possibilità altre, dettate da altre premesse progettuali, ma auspicò che non si demolisse anche la moschea dei bastioni suscitando con la sua proposta interesse e sostanziale consenso (Micacchi, 1934).

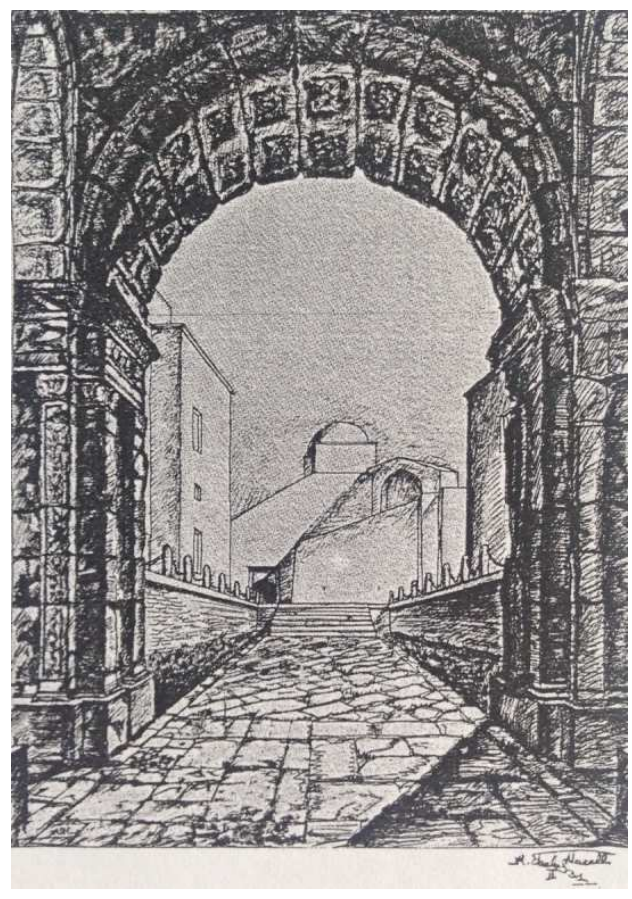

Fig. 5. La Moschea dei Bastioni vista dall'Arco, sistemato, di Marco Aurelio. Disegno di Michele Marelli (Marelli, 1933).

\subsection{Vedute comuni}

La veduta della moschea inquadrata nella cornice di uno dei fornici dell' arco romano di Marco Aurelio (Fig. 5) fu tratteggiata (1931) e argomentata (1933) mentre era in corso la redazione del piano regolatore affidata, lo precisa con ottimismo lui stesso, a "sagaci e competenti colleghi” (Marelli, 1933).

Con Alberto Alpago Novello e Ottavio Cabiati, ma anche con altri come è noto, egli aveva da poco collaborato; in primis per la stesura della apprezzata ma "perdente" proposta per il piano regolatore di Milano.

Ugualmente formatosi al Politecnico, ma più tardi (conseguì la laurea nel 1922), legato in sodalizio con professionisti di indiscussa riconoscibilità (Tomaso Buzzi, Giò Ponti, Emilio Lancia, ...) Marelli è tra quelle figure che impongono di guardare all'attività degli architetti ed archeologi italiani nelle "colonie d'oltremare" oltre il filtro della propaganda e delle matrici più persistenti della storiografia della città. Per alcuni architetti "moderni" infatti, la redazione di un piano urbano o la sistemazione di un isolato mo- 
numentale furono occasioni per riflettere sui valori artistici e storici più diffusamente presenti nella città oltre che sui caratteri costruttivi locali. Non si trattò solo di includerli nella nota riflessione sulla direzione che l'architettura moderna e il loro stesso praticarla, doveva intraprendere, ma anche di assimilare il passato nel presente esercitandosi in un contesto particolare (le colonie) in cui l'identità nazionale che aveva a lungo supportato la cultura del progetto non era del tutto efficace.

\section{Conclusioni}

La demolizione parziale delle mura di Tripoli, risalente ai primi anni dell'occupazione italiana della Libia (1913-15), documenta scarsa attenzione per il passato della città, soprattutto quanto non di età romana. Ma negli anni successivi l'attenzione cambia.

Le accuse di Pietro Romanelli (1923) mostrano una riflessione più matura: egli riconosce più articolata la storia locale e ricorre ad un metodo di lettura archeologica del costruito esteso all'analisi degli elevati. Anche l'approccio al restauro cambia: si mira ad affinare le tecniche di consolidamento, il rispetto delle diverse fasi costruttive, la conservazione tanto degli aspetti formali che materiali. Nel 1930 la redazione di un nuovo piano regolatore verrà così discussa all'interno di un circolo culturalmente ed ideal- mente affiatato interessato a precisare la relazione tra centro storico e nuovi quartieri o il significato della tradizione nell' architettura e nella cultura contemporanea.

\section{Note}

${ }^{1}$ La Tripolitania, oggi parte della Libia, si identifica come la regione litoranea, compresa tra il Golfo di Gabes e il Grande Sirte.

${ }^{2}$ L'Italia aveva dichiarato guerra alla Turchia il 29 settembre del 1911. Pochi giorni dopo (3 ottobre) attaccava il forte "spagnolo" di Tripoli guadagnandola in soli giorni ed insediandosi nel Castello a governarla il 7. Circa un mese dopo (5 novembre) la città fu decretata italiana; anche se la conversione in legge del decreto sarà successiva di qualche mese (25 febbraio 1912).

${ }^{3}$ Boni aveva proposto "una via comoda attraverso le rovine di Oea [Tripolo n.d.R]" che, approfittando della strada che dall' Arco di Marco Aurelio, primo monumento che avrebbe dovuto vedersi allo sbarco, conduceva all'Alto del Forte della Lanterna, già destinato ad ospitare il $\mathrm{Mu}$ seo Tripolitano, avrebbe permesso di attraversare un' area ricca di memorie topografiche e storiche fiancheggiando la chiesa di S. Lazzaro (Boni-Mariani, 1915 p. 34).

\section{Bibliography}

Aurigemma, S. (1915). "Notizie archeologiche sulla Tripolitania", Notiziario Archeologico, I, I-II, pp. 37-64.

Aurigemma, S. (1930). "Federico Halbherr e la missione archeologica italiana in Cirenaica e in Tripolitania", in Africa Italiana, IX, III, 3-4, pp. 237-250.

Aurigemma, S. (1931). "Note al programma per l'esplorazione archeologica della Tripolitania", Rivista delle Colonie Italiane, $\mathrm{V}, 7$, pp. 527-533.

Bartoccini, R. (1924). "Restauri al castello di Tripoli”, Bollettino d'Arte, IV, VI, pp. 279-284.

Belotti, B. (1987). "De Finetti Giuseppe”, in Dizionario biografico degli Italiani, vol. 33.

Boni, G.; Mariani, L. (1915). "Relazione intorno al consolidamento ed al ripristino dell'arco di M. Aurelio in Tripoli", Notiziario Archeologico, I, I-II, pp. 13-34.

Fabiani, E. (1991). "Ottavio Cabiati pittore e incisore", in Cajani, F., ed., Ottavio Cabiati e il suo tempo (1991). Catalogo della mostra (Seregno, Sala Civica Ezio Mariani, 9-20 novembre 1991), Besana Brianza, pp. 73-100.

Gallimberti, N. (1933). "Il piano regolatore di Verona", Urbanistica, II, (2).

Giovannoni, G. (1932). "Nuovi sviluppi dell'urbanistica in Italia”, Urbanistica, I, 6.

Giovannoni, G. (1934). "L'urbanistica italiana alle soglie dell'anno”, Urbanistica, III, 1, pp. 3-9.

Grisoni, M.M. (2016). "Sul progetto di restauro. Note dai disegni di Alessandro Minali (1888-1960)", in Le ragioni del disegno. Pensiero, forma, e modello nella gestione della complessità. $38^{\circ}$ Convegno internazionale dei docenti delle discipline della rappresentazione, Firenze 15-17 settembre 2016.

Lanzani, A. (1991). "L'idea di città e di urbanistica di Ottavio Cabiati", in Cajani, F., ed., Ottavio Cabiati e il suo tempo. Catalogo della mostra (Seregno, Sala Civica Ezio Mariani, 9-20 novembre 1991), pp. 145-178. 
Marchetti, V. (1931). "Il volto della Tripoli nuova", Giornale d'Italia, pp. 1-2.

Marelli, M. (1932). "Relazione intorno al progetto di sistemazione dell'Arco di Marco Aurelio", Africa Italiana, V, 3-4, pp. 162-171.

Mariani, L. (1915). "Prefazione”, Notiziario Archeologico, I, I-II, pp. 7-12.

Micacchi, R. (1934). "L'Arco di Marco Aurelio in Tripoli e la sistemazione della zona adiacente”in Rivista delle colonie italiane, VIII, 10, pp. 824-839.

Ornato, G.Z. (1932). "Nuove opere di rinnovamento a Tripoli”, Regime fascista, 2 March, p. 4.

Palatini, G. (1935). "Urbanistica nei centri di cura, soggiorno e turismo", Urbanistica, IV, 4, pp. 264-265.

Rege, M. De. (1934). "Il nuovo piano regolatore di Tripoli”, Urbanistica, III, 3, pp. 121-128.

Romanelli, P. (1923). "Restauri alle mura barbaresche di Tripoli”, Bollettino d'Arte, II, XII, pp. 570-576.

Torres, D. (1936). "Concezioni dei tracciati di piani regolatori coloniali”, Urbanistica, V, 2, pp. 70-72.

Tripoli e dintorni. Piccola guida della Tripolitania settentrionale. (1914). Minitero delle Colonie, Roma.

Vistoli, F. (2017). "Romanelli, Pietro", in Dizionario biografico degli Italiani, 88.

Zanella, F. (1991). “L'archivio Alpago Novello presso il centro studi e Archivio della Comunicazione dell’Università di Parma", in Cajani, F., ed., Ottavio Cabiati e il suo tempo. Catalogo della mostra (Seregno, Sala Civica Ezio Mariani, 9-20 novembre 1991, Besana Brianza, pp. 191-204.

Zanella, F. (2002). Alpago Novello, Cabiati e Ferrazza 1912-1935, Electa, Milano.

Zanella, F. (2006). "Ottavio Cabiati e Alberto Alpago Novello- Lo studio e l'archivio”, Quaderni della Brianza, 166, pp. 177-181.

Zampagni, T. (2006). "L'influenza dell'orientalismo in Ottavio Cabiati e la nuova immagine della città nei piani urbanistici di Bengasi e Tripoli”, Quaderni della Brianza, 166, pp. 171-176. 